\title{
Distribution of harvestmen of the genus Ischyropsalis C. L. Koch (Arachnida: Opiliones) in Poland
}

\author{
Robert ROZWALKA*, Andrzej MAZUR** and Wojciech STARĘGA*** \\ *Department of Zoology, Maria Curie-Sklodowska University, Akademicka 19, 20-033 Lublin; \\ e-mail: arachnologia@wp.pl \\ **Chair of Forest Entomology, Poznań Life Sciences University, Wojska Polskiego 71c, 60-625 Poznań; \\ e-mail: andrzejm@up.poznan.p \\ ***Institute of Biology, University of Natural History and Humanistics, Prusa 12, 08-110 Siedlce; \\ e-mail: wojstar@op.pl
}

\begin{abstract}
Based on information from literature and new materials the distribution of the genus Ischyropsalis in Poland was studied. New data about $I$. hellwigi and I. manicata expand also information on habitat of these species and their vertical ranges.
\end{abstract}

Key words: Ischyropsalis hellwigi, Ischyropsalis manicata, vertical distribution, identification

\section{INTRODUCTION}

The genus Ischyropsalis C. L. Koch, 1839 is represented in the Polish fauna by two taxa: Ischyropsalis hellwigi hellwigi (Panzer, 1794) and Ischyropsalis manicata L. Koch, 1869 (Staręga 1976). Both species are very characteristic and easy to identify (Figs 1-2), but as they are notoriously difficult to find due to their soil-dwelling habits and presumed rarity their present distributions in Poland are poorly known. This actually applies to the postglacial relict I. hellwigi (Martens 1969, 1978, Staręga 1976), mentioned in Poland so far as several specimens from only few localities in Karkonosze (Riesengebirge) and other parts of Sudeten Mountains and isolated localities in Cracow-Częstochowa Upland (Rafalski 1961, Martens 1969, Sanocka-Woloszyn 1973, 1981, Staręga 1976, Rozwatka 2010). The distribution of I. manicata was known somewhat better. This species has been reported from nearly 20 localities in the Carpathians: from Silesian Beskides, through Tatra to Bieszczady Mountains (Starega 1976, Martens 1978). The present study summarizes information about the occurrences of $I$. hellwigi and $I$. manicata in Poland based on data from literature. Additionally, new material collected mostly by the authors with pitfall traps (2005-2010) and by hand searching (1986-2011) is included.

\section{DIAGNOSIS OF GENUS ISCHYROPSALIS}

Body egg-shaped, uniformly black or brownish black (occasionally greyish). Dorsum mostly with scutum laminatum or scutum parvum (rarely scutum intermedium). Eyes separated by distinct furrow. Chelicerae very massive, distinctly longer than body, with numerous spines. Males with dorsal or dorsomedial field covered by setae in distal part of basal segment ("brush"). Female chelicerae provided with spines only. Pedipalps fairly long and thin. Legs of medium length, quite strong, unarmed. Coxa II-IV not adnate. Labium separated from sternum by distinct furrow, sternum small, about same size as labium. Penis club-shaped, glans clearly separated by often very numerous setae, stylus bent backwards. Ovipositor very short, not 
segmented, with few setae in apical part, receptaculum seminis with numerous (4-10) fingerlike ampullae, basally surrounded with funnel-shaped structure.

Copulative apparatus and other morphological characteristics of both species are fully described and illustrated in the papers of Šilhavý (1956), Martens (1969, 1978) and Staręga (1976).

\section{KEY TO POLISH SPECIES}

1. Chelicerae with dorsal „brush" on distal part of basal segment males (2)

- Chelicerae without dorsal ,brush" on distal part of basal segment females (3)

2. „Brush"situated on dorsal side only, its length equal to about $1 / 3$ length of this segment

- „Brush" situated on dorsal and mediolateral side, its length equal to about 0.2 length of this segment I. manicata

3. Dorsum with scutum laminatum I. hellwigi

- Dorsum with scutum parvum I. manicata
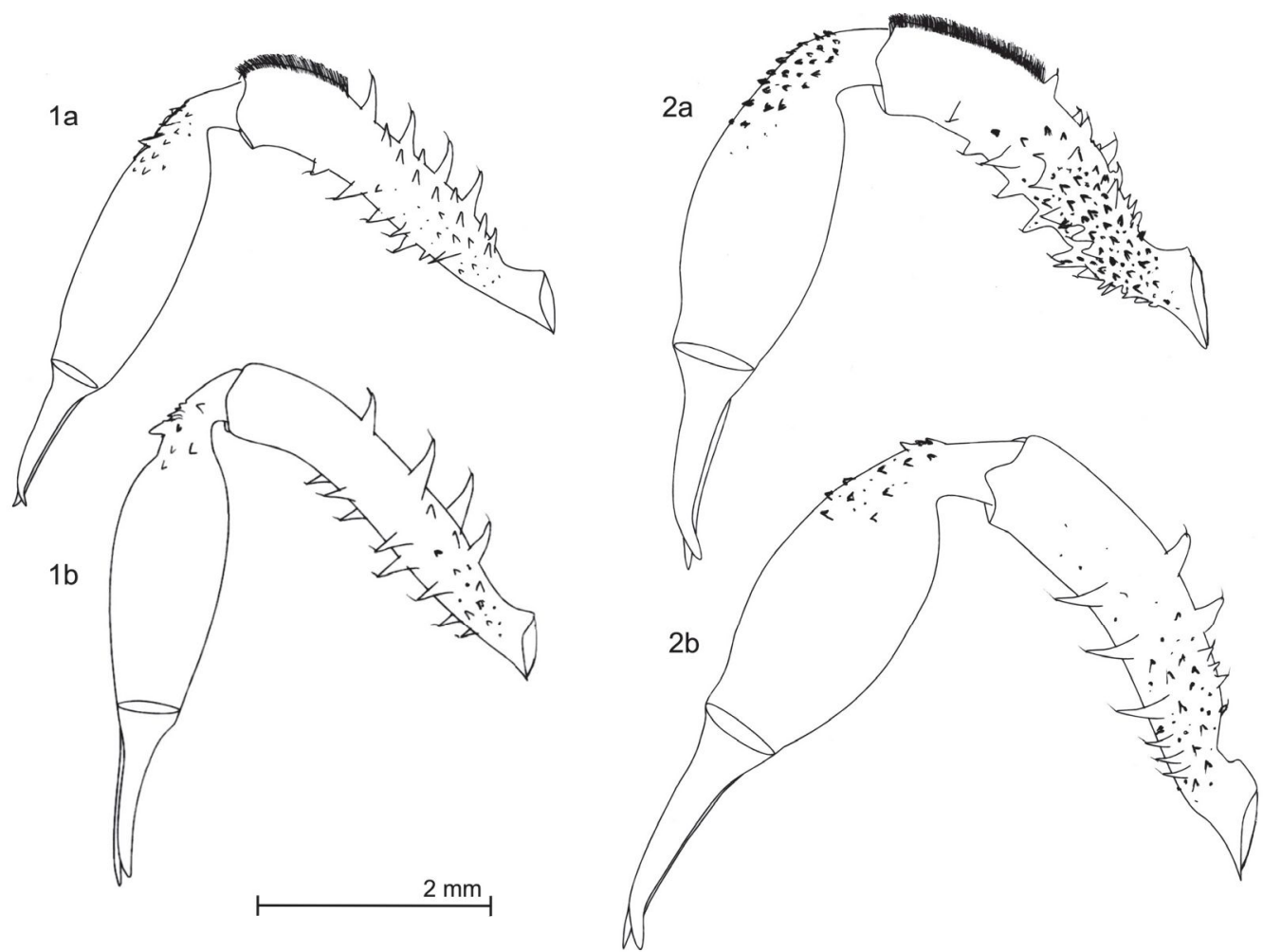

Figs 1-2. Chelicerae of Ischyropsalis, lateral view. 1. I. manicata: $\mathbf{a}-$ male, $\mathbf{b}$-female. 2. I. hellwigi: $\mathbf{a}-$ male, $\mathbf{b}-$ female. 


\section{Ischyropsalis hellwigi hellwigi (Panzer, 1794)}

General distribution. Plains, foothills and medium-high mountains of Central Europe: S Netherlands, S and W Germany, W and C Bohemia, Austria, W Hungary, Croatia and Bosnia (Martens 1965, 1978, Novak 2005, Wijnhoven 2009). The second subspecies: I. hellwigi lucantei Simon, 1879, occurs in the Pyrenees (Martens 1969, 1978).

Recently appeared information about the occurrence of $I$. hellwigi in Southern Turkey (Kurt et al. 2008, Bayram et al. 2010 - as I. taunica Müller, 1923). The accompanying drawings (p. 656, figs 13-14), are very vague, but clearly show this is not Ischyropsalis. The shape and construction of chelicerae, point on a representative of the genus Zachaeus C. L. Koch (Phalangiinae)! Moreover, the distribution of the genus Ischyropsalis is generally considered to be limited to Southern and Central Europe (Martens 1978).

Distribution in Poland. Karkonosze (Riesengebirge) Mountains, other parts of Sudeten, southern part of Cracow-Częstochowa Upland (possibly isolated population - anyway no data from the "in-between" area) - altogether about 10 localities (Grube 1871, Lebert 1875, Fickert 1876, Roewer 1923, Bartoš 1938, Rafalski 1960, 1961, Martens 1969, 1978, SanockaWołoszyn 1973, 1981, Starega 1976, Sanocka 1983, Rozwałka 2010).

New localities (all in Sudeten) (Fig. 3):

1 ₹ : 16.08-6.09.2005, Izerskie Mts, forest insp. Świeradów, forest sec. $358 \mathrm{~d}$, ca $1080 \mathrm{~m}$ a.s.1., upper montane spruce forest at regeneration phase, pitfall traps, leg. A. Mazur, det. R. Rozwalka [WS 23].

1 ठ̊: 15.08-7.09.2005, Karkonosze National Park, protective district Szrenica, forest sec. $201 d$, ca $1200 \mathrm{~m}$ a.s.1., upper montane spruce forest bordering with peat bog spruce forest; pitfall traps, leg. A. Mazur, det. R. Rozwalka [WS 32].

4 ठð: 15.08-21.10.2005, Karkonosze National Park, protective district Przelęcz, forest sec. 139i, ca $1090 \mathrm{~m}$ a.s.1., upper montane compact spruce forest around 90 years old, pitfall traps, leg. A. Mazur, det. R. Rozwalka [WS 42].

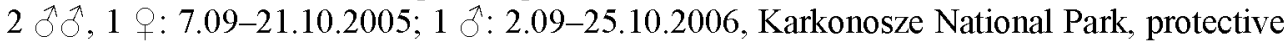
district Przelęcz, forest sec. $150 \mathrm{~g}$, ca $1090 \mathrm{~m}$ a.s.l., upper montane spruce forest in renewal phase (approximately 15 years), pitfall traps, leg. A. Mazur, det. R. Rozwalka [WS 42].

6 of 1 ㅇ: 15.08-7.09.2005, Karkonosze National Park, protective district Przelęcz, forest sec. $150 \mathrm{~h}$, ca $1040 \mathrm{~m}$ a.s.l., upper montane spruce forest decay phase of about 8-year-old brushwood, pitfall traps, leg. A. Mazur, det. R. Rozwalka [WS 42].

1 ㅇ: 8.09-8.10.2005, Śnieżnik Massif, Czarna Góra Mt, forest insp. Międzylesie, forest sec. $115 \mathrm{a}$, ca $1200 \mathrm{~m}$ a.s.l., approximately 5-year-old culture of spruce with clumps of dwarf pine in alpine coniferous forest, pitfall traps, leg. A. Mazur, det. R. Rozwalka [XR 26].

1 juv.: 3.07-31.08.2005, Śnieżnik Massif, Czarna Góra Mt, forest insp. Ladek-Zdrój, forest sec. 339h, ca $1160 \mathrm{~m}$ a.s.l., upper montane spruce forest, pitfall traps, leg. A. Mazur, det. R. Rozwałka [XR 26].

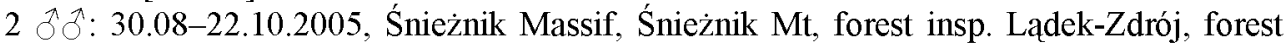
sec. 288c, ca $1230 \mathrm{~m}$ a.s.1., sparse upper montane spruce forest, pitfall traps, leg. A. Mazur, det. R. Rozwałka [XR 36].

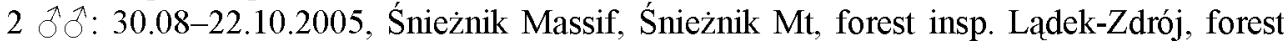
sec. 296a, ca $1230 \mathrm{~m}$ a.s.1., upper montane spruce forest, pitfall traps, leg. A. Mazur, det. R. Rozwałka [XR 36].

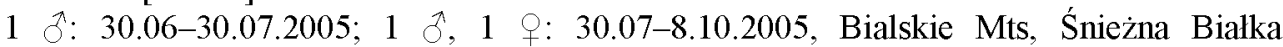
Wilderness; forest insp. Ladek-Zdrój, forest sec. $367 \mathrm{~b}$, ca $980 \mathrm{~m}$ a.s.l., transition zone between lower mountains sycamore-beech forest and upper montane spruce forest, pitfall traps, leg. A. Mazur, det. R. Rozwalka [XR 46]. 


\section{Ischyropsalis manicata L. Koch, 1869}

General distribution. Carpathian species, living in medium-high mountains from the Moravian Gate to the Iron Gate: SE Moravia, S Poland, Slovakia, SW Ukraine, Romania (Šilhavý 1956, 1973, Martens 1969, 1978, Staręga 1976, Stašiov 1999, Bezděčka 2010).

Distribution in Poland (literature data). Silesian Beskides, High Beskides (Babia Góra Mt.), Western and High Tatra, Pieniny Mountains?, Bieszczady Mountains - altogether approximately 18 localities (Nowicki 1869, L. Koch 1870, Roewer 1914, 1923, 1950, Kowalski 1955, Rafalski 1960, 1961, Lomnicki 1963, Staręga 1966, 1976, 1979, Martens 1969, 1978, Sanocka 2003). Starega (1979) believed that the species could become extinct in Pieniny Mts, because, despite intensive search in the 70s of the twentieth century, there was identified a trace of his presence - a single dry chelicera discovered in the $30 \mathrm{~s}$. Martens (1969) also reported Rogóźno near Rybnik as a locality of $I$. manicata. It is, however, doubtful, because at the time the material has been collected (1884, Guzy leg., ex coll. W. Kulczyński) this territory belonged to Germany and could not bear a Polish name. Now it is part of the town Żory (Germ. Sohrau) in Upper Silesia - a region seriously disturbed by the heavy industry [in 1884 the industry already existed and the changes of environment were considerable]. We could not trace any other locality in southern Poland fitting the mentioned name.

New localities (Fig. 3):

$1 \delta:$ 4.07.2009, Western Tatra, Tatra National Park, Ciemniak Mt, under stones in the high mountain pasture a little below the peak, ca 2094-2095 m a.s.1., leg. R. Gosik, det. R. Rozwalka [DV 25].

1 : 6.07.2008, High Tatra, Tatra National Park, Pięć Stawów Polskich Valley, dwarf mountain pine forest, under stone near Siklawa waterfall, ca $1700 \mathrm{~m}$ a.s.1., leg. et det. R. Rozwalka [DV 35].

1 §̧: 2.09.1986, Gorce Mts, Ochotnica Górna-Jaszcze Male, over Jaszcze stream, under stones, ca $850 \mathrm{~m}$ a.s.1., leg. W. Jędryczkowski, det. W. Staręga [DV 49].

1 J : 6.09-7.10.2007, Western Beskid Mts, Żmiącki stream valley, distr. Limanowa, mixed sycamore-beech forest, ca $760 \mathrm{~m}$ a.s.1., pitfall traps, leg. M. Szewczyk, det. R. Rozwalka [DA 60].

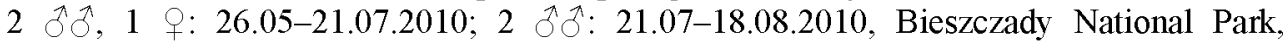
Polonina Caryńska Mt, mountain pasture, ca 1115-1120 m a.s.l., pitfall traps, leg. et det. R. Rozwalka [FV 14].

1 壬 26.05.2010, Bieszczady National Park, Polonina Caryńska Mt, S slope, beech forest, under stone, ca $870 \mathrm{~m}$ a.s.1., leg. et det. R. Rozwałka [FV 14].

1 O : 26.05-21.07.2010, Bieszczady National Park, Połonina Caryńska Mt, S slope, ca 860 $870 \mathrm{~m}$ a.s.l., beech forest, pitfall traps, leg. et det. R. Rozwalka [FV 14].

1 juv.: 27.04.2011, Bieszczady National Park, Bukowe Berdo Mt, NE slope, ca $820 \mathrm{~m}$ a.s.l., beech forest, under stones, leg. et det. R. Rozwalka [FV 24].

$1 \hat{\jmath}: 3-25.05 .2010 ; 1$ : $25.05-18.07 .2010 ; 2 \hat{\jmath}: 18.07-14.08 .2010$, Bieszczady National Park, piedmont peat-bog reserve Tarnawa, swamp pine forest, ca $642 \mathrm{~m}$ a.s.1., pitfall traps, leg. et det. R. Rozwałka [FV 34].

Localities published on maps by Staręga (1976) without documentation:

1 O, 4 q9: 8.06.1967, Beskid Ślaski Mts, below the peak Mala Czantoria, sycamore-spruce forest, in leaf litter and under stones, ca $700 \mathrm{~m}$ a.s.1., leg. A. Riedel, det. W. Staręga [CA 40].

1 : 6.08.1964, Middle Beskid Mountains, Rytro near Nowy Sącz, Wielka Roztoka Valley, beech forest, in litter, leg. et det. W. Staręga [DV 78]. 


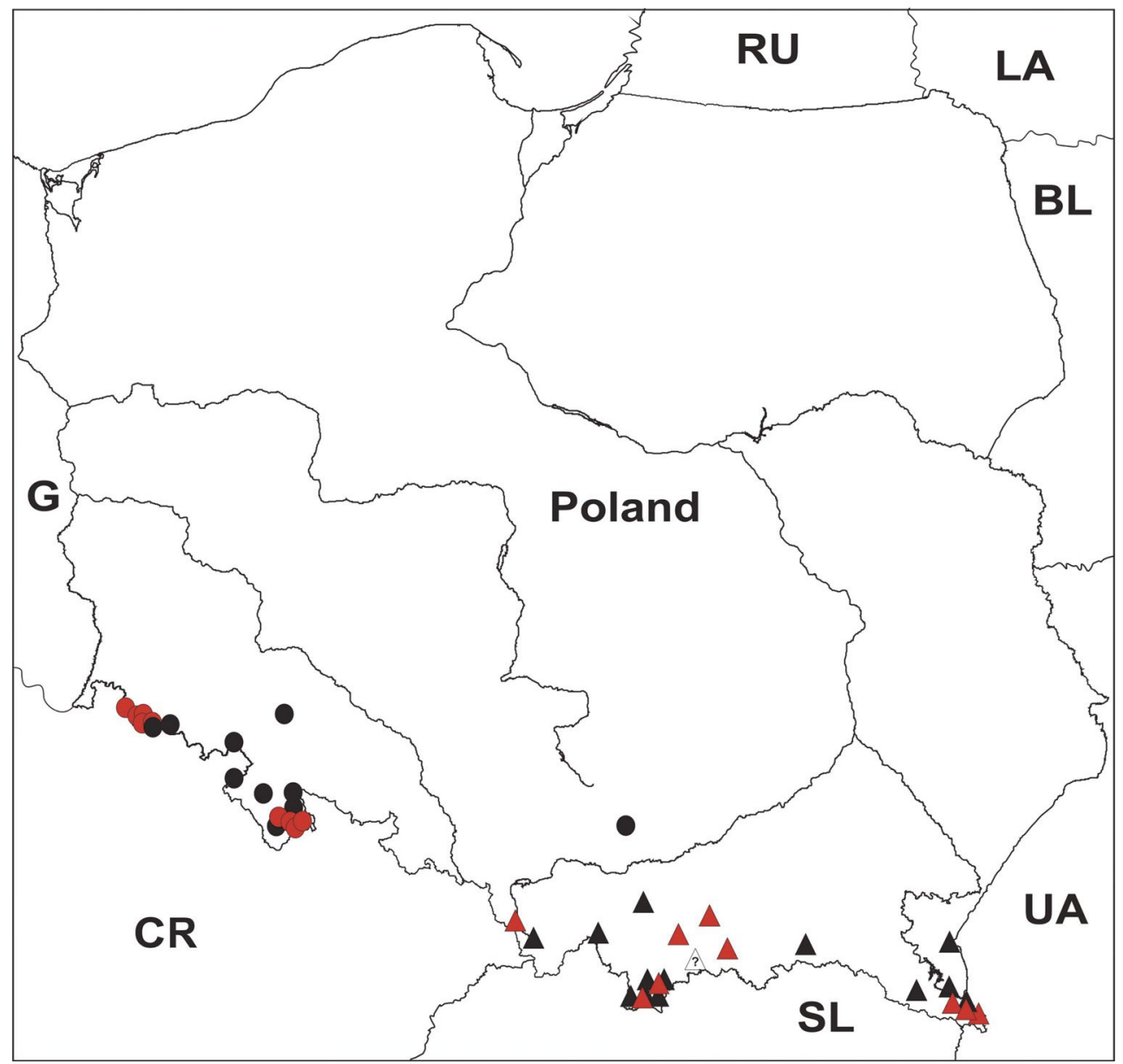

Fig. 3. Distribution of Ischyropsalis in Poland: circles - I. hellwigi, triangles $-I$. manicata; black - literature data, red - new records.

\section{HABITAT, PHENOLOGY AND FOOD PREFERENCES}

Ischyropsalis hellwigi is a postglacial relict species, occurring most often in environments characterized with low constant temperature and high humidity (Martens 1965, 1978, Starega 1976). This species is mostly caught in a cool mountain valleys and foothill streams under moist, rotting logs of wood, stones, in moist leaf litter or in caves (Martens 1965, 1969, 1978, Sanocka-Wołoszyn 1973, 1981, Staręga 1976, Bliss 1980, Baehr \& Baehr 1985). Vertical distribution of this species covers the range from about $50 \mathrm{~m}$ above sea level in the Netherlands to $1900 \mathrm{~m}$ a.s.l. in the Austrian Alps (Martens 1978). In Poland the published records range from 250 to $650 \mathrm{~m}$ a.s.1. (Starega 1976). According to Martens $(1969,1978)$ it is a stenochronic species, occurring from late August to early November. According to Staręga (1976) it is as eurychronic species (April-December). The exclusive food source of these harvestmen are shell snails: Zonitidae, Helicidae, Clausiliidae as well as shell-less slugs: Limacidae, Arionidae (Verhoeff 1900, Martens 1969, 1978, Staręga 1976). 
Ischyropsalis manicata occurs from lower forest zone to dwarf mountain pine, about 300 to about $1700 \mathrm{~m}$ a.s.l. (Staręga 1976, Martens 1978, Stašiov 1999, 2008). Like I. hellwigi, I. manicata was mainly caught in the valleys of streams, in damp, rotting wood logs, under rocks or in thick forest litter (Starega 1976, Martens 1978, Sanocka 2003). In the Romanian Carpathians and Tatra Mountains the species was recorded from caves (Kowalski 1955, Avram 1964, Sanocka 2003). Adults occur over the whole year (Starega 1976). Food preferences are probably similar to $I$. hellwigi, but because of the smaller body size (I. manicata: $\hat{\delta}=4.5-6.0$, $\phi=7.0-7.8 \mathrm{~mm} ;$ I. hellwigi: $\hat{\delta}=5.6-6.8$, $q=6.8-8.5 \mathrm{~mm}), I$. manicata may feed on comparatively smaller and more delicate shell snails (Martens 1965, 1978).

\section{CONCLUSIONS}

The present data supplement the information about occurrence of $I$. hellwigi and I. manicata in Poland. Particularly valuable are new localities of $I$. hellwigi found on the area of Karkonosze and other chains of Sudeten Mountains. Most data about this very rare species come from the time of second half of XIX century and beginnings of XX century (Lebert 1875, Fickert 1876, Roewer 1923, Bartoš 1938). Since those publication there were very few recent data confirming the presence of this species in Sudeten (Rafalski 1961, Starega 1976, Sanocka 1983) and in Cracow-Częstochowa Upland (Sanocka-Woloszyn 1973). Moreover, the present localities of I. hellwigi move its vertical range in Poland from about $650 \mathrm{~m}$ to about $1230 \mathrm{~m}$.

Our new findings on the vertical distribution of 1 . manicata are also of particular interest. So far, the highest reported findings were from Babia Góra Mountain (about $1700 \mathrm{~m}$ ) (Rafalski 1961), Źólta Turnia Massif in the High Tatra Mountains (Łomnicki 1963) and from caves in the Western Tatra (Kowalski 1955, Sanocka 2003). Information from Slovakia (Stašiov 2008) and Romania (Avram 1964, Martens 1978) shows a similar maximum altitude reached by this species. Specimen caught in the stone rubble just beneath the peak of Ciemniak (Western Tatra), moves the border of vertical range to almost $2100 \mathrm{~m}$ a.s.1. Furthermore, our new data demonstrate that $I$. manicata, traditionally considered associated with mountain and submountain forests (or dwarf mountain pine forest), also penetrates into the high alpine pasture zone. Occurrence of 1 . manicata above the upper border of mountain forests is also confirmed by observations in the Bieszczady Mountains, where this species was recorded in the upper parts of Polonina Caryńska (1120 m a.s.1.).

\section{REFERENCES}

AvRAM S. 1964. Observations sur la variabilité de l'espèce Ischyropsalis dacica Roewer des grottes de Roumanie. Revue Roumaine de Biologie, Série de Zoologie 9: 245-256.

BAEHR M., BAEHR B. 1985. Zur Verbreitung und Biotopwahl des Schneckenkankers Ischyropsalis hellwigi Panzer im Schönbuch bei Tübingen (Opiliones, Ischyropsalidae). Jahreshefte der Gesellschaft für Naturkunde 140: $203-212$.

BARTOŠ E. 1938. Die Ischyropsaliden der Tschechoslowakischen Republik. Zoologischer Anzeiger 123: $155-159$.

BAYRAM A., ÇORAK İ., DANIŞMAN T., SANCAK Z., YIĞIT N. 2010. Checklist of the harvestmen of Turkey (Arachnida: Opiliones). Munis Entomology \& Zoology 5: 563-585.

BEZDĚČKA P. 2010. Sekáči (Opiliones) Bílých a Bielych Karpat. In: TRAVNIČEK D., ŠUŠOLOvÁ J. Západné Karpaty společná hranica. Sborník prríspěvkủ z II. Mezinárodního sympózia přírodovĕdcủ Trenčianského kraju a Zlínského kraje, 9-11.VI.1010, pp. 47-54. Zlin (Muzeum jihovýchodní Moravy), 112 pp.

BLISS P. 1980. Zur Ökologie und Verbreitung des Schneckenkankers, Ischyropsalis hellwigi hellwigi, in der DDR (Opiliones, Ischyropsalididae). Hercynia, N.F. 17: 292-302.

Bliss P., BLICK T., RAUSCH H., MALTEN A. 1992. Rote Liste gefährdeter Weberknechte (Opiliones) Bayerns. Landesamt für Umweltschutz 111: 67-68.

FICKERT C. 1876. Myriopoden und Araneiden vom Kamme des Riesengenbirges. Ein Beitrag zur Faunistik der Subalpinen Region Schlesiens. Zeitschr. für Entomologie, Breslau, 2: 1-48.

GRUBE E. 1871. Über die schlesische Arachnidenfauna. Jahresberichte Schlesischer Gesellschaft Vaterländischer Cultur 48: 86-87. 
KLIMEŠ L. 2005. Opiliones (sekáči). In: FARKAČ J, KRÁL D., ŠKORPÍK M. (eds), Červený seznam ohrožených druhů České republiky. Bezobratlí [Red list of threatened species in the Czech Republic. Invertebrates], pp. 85-87. Agentura ochrany prírody a krajiny $\breve{C} R$, Praha, 760 pp.

KocH L. 1870. Beiträge zur Kenntniss der Arachnidenfauna Galiziens. Jahrbücher kaiserlich-königlicher Gelehter Gesesellschaft 41, [Sep.] 56 pp.

KoMPosch C. 1999. Rote Liste der Weberknechte Kärntens (Arachnida: Opiliones). Naturschutz in Kärnten 15: $547-565$.

KOWALSKI K. 1955. Fauna jaskiń Tatr Polskich. Ochrona Przyrody 23: 283-332.

KuRT K., BABAŞOĞLU A., SEYYAR O., DEMIR K., TOPÇU A. 2008. New faunistic records for the Turkish harvestmen fauna (Arachnida: Opiliones). Munis Entomology \& Zoology 3: 654660

LEBERT H. 1875. Verzeichnis der Schlesischen Spinnen mit Aufzählung der schlesischen Myriapoden. Tübingen, $63 \mathrm{pp}$.

LOMNICKI A. 1963. The distribution and abundance of ground-surface-inhabiting arthropods above the timber line in the region of Źólta Turnia in the Tatra Mts. Acta Zoologica Cracoviensia 8: 183-249.

MARTENS J. 1965. Verbreitung und Biologie des Schneckenkankers Ischyropsalis hellwigi. Natur und Museum 95 : 143-149.

MARTENS J. 1969. Die Abgrenzung von Biospezies auf biologisch-ethologischer und morphologischer Grundlage am Beispiel der Gattung Ischyropsalis C. L. Koch 1839 (Opiliones, Ischyropsalididae). Zoologische Jahrbücher Systematik 96: 133-264.

MARTENS J. 1977. Rote Liste gefährdeter Tierarten in der Bundesrepublik Deutschland, II - Wirbellose; 3 Weberknechte Opiliones (Spinnentiere). Natur und Landschaft 52: 148-149.

MARTENS J. 1978. Spinnentiere, Arachnida. Weberknechte, Opiliones. Die Tierwelt Deutschlands, 64. Jena (Gustav Fischer Verl.), 464 pp.

NOVAK T. 2005. An overview of Harvestmen (Arachnida: Opiliones) in Bosnia and Herzegovina. Natura Croatica 14: $301-350$

NOWICKI M. 1869. Zapiski faunicze. Sprawozdanie Komisyi Fizyograficznej 3: 145-152.

RAFALSKI J. 1960. Kosarze (Opiliones). Katalog Fauny Polski, 32, 2. PWN, Warszawa, 29 pp.

RAFALSKI J. 1961. Prodromus faunae Opilionum Poloniae. Prace Komisji Biologicznej Poznańskiego Towarzystwa Przyjaciól Nauk 25: 325-372.

ROEWER C.F. 1914. Die Familien der Ischyropsalididae und Nemastomidae der Opiliones-Palpatores. Archiv für Naturgeschichte 80A, 3: 99-169.

ROEWER C.F. 1923. Die Weberknechte der Erde. Systematische Bearbeitung der bisher bekannten Opiliones. Gustav Fischer Verl., Jena, $1116 \mathrm{pp}$.

ROEWER C.F. 1950. Über Ischyropsalidide und Trogulidae (Weitere Weberknechte XV). Senckenbergiana 31: 11-50.

ROzWAŁKA R. 2010. Ischyropsalis hellwigi (Panzer, 1794) (Arachnida: Opiliones) w Parku Narodowym Gór Stołowych. Przegląd Zoologiczny 52 (2008): 167-170.

SANOCKA E. 1983. Kosarze (Opiliones) strefy przełomów pod Książem (woj. walbrzyskie). In: GLOwACKAMigula E., Skowerska M., WoJCIECHOWski W. (eds), Postępy zoologii (Materiały informacyjne na XIII Zjazd Polskiego Towarzystwa Zoologicznego. Katowice, wrzesień 1983, p. 124. Wydawnictwo Uniwersytetu Śląskiego, Katowice.

SANOCKA E. 2003. Kosarze (Opiliones) masywu Babiej Góry. In: WoŁoszyn B.W., WoŁOSZYN D., CELARY W. (eds), Monografia fauny Babiej Góry, pp. 103-118. Publikacje Komitetu Ochrony Przyrody PAN, Kraków.

SANockA-WokoszYN E. 1973. Ischyropsalis hellwigi (Panzer, 1794) (Opiliones) z Ojcowskiego Parku Narodowego. Przegląd Zoologiczny 17: 49-52.

SANOCKA-WOEOSZYN E. 1981. Badania pajęczaków (Aranei, Opiliones, Pseudoscorpionida) Wyżyny KrakowskoCzęstochowskiej. Acta Universitatis Wratislaviensis 548, Prace Zoologiczne 11, 92 pp.

ŠILHAVÝ V. 1956. Sekáči - Opilionidea. Fauna ČSR, 7. Akademie Věd, Praha, 272 pp.

ŠILHAvÝ V. 1973. Prvý nález sekáče Ischyropsalis manicata L. Koch na Moravě. Sborník Př́rodovědný Západomoravského Muzea 9: 83-84.

STARĘGA W. 1966. Kosarze (Opiliones) Bieszczad. Fragmenta Faunistica 13: 145-157.

STAREGA W. 1976. Kosarze - Opiliones. Fauna Polski, 5. Państwowe Wydawnictwo Naukowe, Warszawa, 197 pp.

Starega W. 1979. Kosarze (Opiliones) Pienin. Fragmenta Faunistica 24: 175-183.

StaręGa W., Blaszak C., RAFAlski J. 2002. Arachnida Pajęczaki. In: Glowactński Z. (ed.), Czerwona lista zwierząt ginących i zagrożonych w Polsce, pp. 133-140. Instytut Ochrony Przyrody PAN, Kraków, 155 pp.

STAŠIOV S. 1999: Distribution of Ischyropsalis manicata (Opilionida) in Slovakia. Entomofauna Carpathica 11: 9-12.

STAŠIOV S. 2008. Altitudinal distribution of harvestmen (Euchelicerata: Opiliones) in Slovakia. Polish Journal of Ecology 56: 157-163.

VERHOEFF C. 1900. Zur Biologie von Ischyropsalis. Zoologischer Anzeiger 23: 106-107.

WisNHOvenN H. 2009. De Nederlandse hooiwagens (Opiliones). Entomologische Tabellen 3: 118 pp. 
[Rozmieszczenie kosarzy z rodzaju Ischyropsalis C. L. Koch (Arachnida: Opiliones) w Polsce]

Praca omawia dotychczas znane rozmieszczenie i nowe stanowiska Ischyropsalis hellwigi hellwigi i $I$. manicata w Polsce. Ten pierwszy jest w kraju zwiazany z Sudetami i Jura Krakowsko-Częstochowska, drugi - z Karpatami, od Beskidu Śląskiego po Bieszczady. Nowe znaleziska znacznie rozszerzaja pionowy zasięg obu gatunków: w przypadku $I$. hellwigi do $1230 \mathrm{~m} \mathrm{n.p.m}$. (dotychczas bylo $650 \mathrm{~m}$ ), a w przypadku $I$. manicata do prawie $2100 \mathrm{~m} \mathrm{n.p.m}$. (dotychczas okolo $1700 \mathrm{~m}$ ). 